\title{
Anorectal dysfunction in multiple sclerosis patients: A pilot study on the effect of an individualized rehabilitation approach
}

\author{
Martina Kovari $^{\mathrm{a}}$, Jan Stovicek ${ }^{\mathrm{b}}$, Jakub Novak ${ }^{\mathrm{a}, *}$, Michaela Havlickova ${ }^{\mathrm{a}}$, Sarka Mala ${ }^{\mathrm{b}}$, \\ Andrew Busch ${ }^{\mathrm{c}}$, Pavel Kolar ${ }^{\mathrm{a}}$ and Alena Kobesova ${ }^{\mathrm{a}}$ \\ ${ }^{a}$ Department of Rehabilitation and Sports Medicine, Second Faculty of Medicine, Charles University \\ and University Hospital Motol, Prague, Czech Republic \\ ${ }^{\mathrm{b}}$ Department of Internal Medicine, Second Faculty of Medicine, Charles University and University Hospital \\ Motol, Prague, Czech Republic \\ ${ }^{\mathrm{c}}$ Department of Health and Human Kinetics, Ohio Wesleyan University, Delaware, OH, USA
}

Received 5 August 2021

Accepted 19 October 2021

\begin{abstract}
.
BACKGROUND: Anorectal dysfunction (ARD), especially bowel incontinence, frequently compromises the quality of life in multiple sclerosis (MS) patients. The effect of rehabilitation procedures has not been clearly established.

OBJECTIVE: To determine the effect of an individualized rehabilitation approach on bowel incontinence and anorectal pressures.

METHODS: MS patients with ARD underwent 6-months of individually targeted biofeedback rehabilitation. High resolution anorectal manometry (HRAM) and St. Mark's Fecal Incontinence Scores (SMIS) were completed prior to rehabilitation, after 10 weeks of supervised physiotherapy, and after 3 months of self-treatment.

RESULTS: Ten patients (50\%) completed the study. Repeated measures analysis of variance (ANOVA) demonstrated significant improvement in the SMIS questionnaire over time [14.00 baseline vs. 9.70 after supervised physiotherapy vs. 9.30 after self-treatment $(p=0.005)]$. No significant improvements over time were noted in any HRAM readings: maximal pressure [ $49.85 \mathrm{mmHg}$ baseline vs. 57.60 after supervised physiotherapy vs. 60.88 after self-treatment $(p=0.58)$ ], pressure endurance [36.41 vs. 46.89 vs. $49.95(p=0.53)$ ], resting pressure [55.83, vs 52.69 vs. $51.84(p=0.704)$ ], or area under the curve [230.0 vs. 520.8 vs. $501.9(p=0.16)]$.
\end{abstract}

CONCLUSIONS: The proposed individualized rehabilitation program supports a positive overall effect on anorectal dysfunction in MS patients.

Keywords: Multiple sclerosis, bowel incontinence, high resolution anorectal manometry, St. Mark's fecal incontinence score, biofeedback

\footnotetext{
*Address for correspondence: Jakub Novak, PT, Department of Rehabilitation and Sports Medicine, Second Medical Faculty, Charles University and University Hospital Motol, V Uvalu 84, Prague 5, 150 06, Czech Republic. Tel.: +420 22443 9264; Fax: +420 22443 9220; E-mail: kuba-novak@ seznam.cz.
}

\section{Introduction}

Multiple sclerosis (MS) is one of the most common neurological diseases affecting pelvic floor $(\mathrm{PF})$ and gastrointestinal tract function including defecation (Krogh \& Christensen, 2009; Nusrat et al., 2012; Preziosi \& Emmanuel, 2009). Anorectal dysfunction (ARD) resulting from gastrointestinal autonomic 
disturbance is a frequent symptom in the multiple sclerosis population (Pinter et al., 2015). Compromised coordination of the anorectal musculature may cause many symptoms such as difficult defecation, incontinence, pelvic organs prolapse and pelvic or perianal pain during defecation. These symptoms can occur both separately, and in various combinations (Preziosi \& Emmanuel, 2009).

The incidence of ARD symptoms are broad, ranging from $48 \%$ (Munteis et al., 2006) to $68 \%$ (Hinds et al., 1990), which is perhaps due to a lack of patient reporting, or specific questioning by clinicians. These symptoms tend to increase with age, disease duration, coincidence of urinary dysfunctions, and progressing disability score (Munteis et al., 2006). Females seem to be more frequently affected, especially during menopause hormonal changes, or due to episiotomy or labor trauma. ARD is more common in the primary progressive form of MS (Pinter et al., 2015). The prevalence of ARD is higher with progressive worsening of functional status, however, it can occur at any stage of the disease (Nusrat et al., 2012; Preziosi \& Emmanuel, 2009). MS patients experience constipation in 18-43\%, and stool incontinence in $3-51 \%$ (Nusrat et al., 2012) with both symptoms often coexisting (Wiesel, 2000). Coincidence of ARD with urinary dysfunction is also frequently reported (Chia et al., 1995).

Pathophysiology of ARD in MS patients is quite complex, since MS may impair stool continence and defecation at all levels of central nervous processing. Stool incontinence may result from abnormal peristalsis, anorectal hyposensitivity, anal sphincter weakness, prolonged rectoanal inhibitory reflex, impaired rectal compliance or loss of voluntary control of defecation (Preziosi \& Emmanuel, 2009). Constipation is even more multifactorial with prolonged colonic transit, abdominal wall weakness, anorectal hyposensitivity, PF dyssynergia, poor diet and behavioral factors such as previous episodes of fecal incontinence and problem to access the toilet playing the role (Preziosi \& Emmanuel, 2009). Overflow incontinence is associated with long-term constipation, when sebum is formed in the rectum, resulting in liquid stool and fluid leaks (Lensch \& Jost, 2011). MS patients often report limited movement activities (Motl et al., 2017). Lack of movement adversely affects muscle function, including the PF muscles. Patients further reduce mobility to prevent stool and urine leakage out of the house. Hypomobility is related to obesity a reduced muscle power. Thus, the vicious circle is formed. The clinical picture of the ARD is variable. The symptoms can combine, progress and change. Both the constipation and the stool incontinence have negative impact on patient's quality of life (Nusrat et al., 2012).

ARD needs to be assessed and treated comprehensively including physiotherapeutic intervention addressing PF dysfunction (Ruiz \& Kaiser, 2017). Conservative treatment affecting behavioral aspects, adequate diet and fluid intake, biofeedback and defecation reflex training as well as regular physical activity are reported to be beneficial (Bywater \& While, 2006; Preziosi et al., 2018). Despite the high prevalence of ARD in MS, exact treatment guidelines are still not available. The treatment is mostly empirical and individually tailored based on patient's actual symptoms and treatment preferences (Preziosi et al., 2018). Physiotherapy trains the awareness of the PF muscles, the selective contraction and relaxation of the anal sphincter and PF (Bols et al., 2007) aiming to restore adequate tone and coordination of the PF musculature (Ruiz \& Kaiser, 2017), and to improve sensitivity of the rectum (Pedraza et al., 2014). Endurance training may help to reduce bowel urgency and the number of incontinence episodes (Pedraza et al., 2014). The problem needs to be explained to the patient in detail. Description of the basic pelvic anatomy, therapeutic goals and time needed for the training to achieve the goals may help to motivate the patient (Pedraza et al., 2014). Cognitive deficit prevents effective physiotherapy (Beer et al., 2012).

Both, subjective and objective assessment is necessary to set up optimal treatment strategy. Various scores and subjective questionnaires evaluating the quality of life of patients with incontinence and the incontinence itself, and scoring its severity are available. The questionnaires also serve as a feedback to evaluate the therapeutic results. For the purposes of this study, the St. Mark 's Faecal Incontinence Score questionnaire was used (Maeda et al., 2008). High resolution anorectal manometry (HRAM) measures the anorectal resting and squeeze pressure, squeeze endurance and propulsive force (Gosling et al., 2019; Lee \& Bharucha, 2016). In MS patients impaired pelvic floor coordination (Marola et al., 2016), decreased anal pressure at rest and reduced maximum sphincter pressure often occurs (Munteis et al., 2008; Nordenbo et al., 1996; Waldron et al., 1993). According to Munteis et al. the maximum sphincter pressure is more reduced in patients with more severe disability and primary progressive form of MS (Munteis et al., 2008). 
Table 1

Participants' anthropometric characteristics. $n=10$, all females

\begin{tabular}{lccccc}
\hline & Age (years) & Height $(\mathrm{cm})$ & Weight $(\mathrm{kg})$ & BMI & EDSS \\
\hline Mean & 47.15 & 169.07 & 73.46 & 25.52 & 3.65 \\
SD & 11 & 8.09 & 14.7 & 3.43 & 1.55 \\
Min & 27 & 156 & 55 & 19.3 & 2 \\
Max & 62 & 188 & 109 & 30.84 & 6.5 \\
\hline Note: & SD = Standard deviation, & BMI = body & mass & index, \\
EDSS = Expanded disability status scale. & & &
\end{tabular}

The aim of this study was to analyze HRAM findings in MS patients with ARD, specifically stool incontinence, and to evaluate the effect of individual physiotherapy and self-treatment with biofeedback on stool incontinence episodes, anal sphincter function and the quality of life.

\section{Materials and methods}

\subsection{Participants}

Initially, 20 patients ( 3 males, 17 females) with various types of relapsing-remitting, primary or secondary progressive (RS) reporting unwanted leakage of stool at least once a month were recruited to the study. Only 10 female patients completed the entire study, due to various reasons. Three patients reported low self-treatment compliance, and their third assessment was not performed. Two patients did not finish because they suffered MS relapse resulting in inability to perform regular self-treatment. One patient encountered concurrent infectious disease during the study period. Two patients found HRAM uncomfortable and refused to undergo the HRAM repeatedly, and two patients found regular treatment too challenging and did not follow the prescribed exercise protocol. Written informed consent was obtained from each participant, and demographic characteristics of the sample including gender, age, weight height and MS stage measured by Expanded Disability Status Scale (EDSS) are shown in Table 1. The study conforms with The Code of Ethics of the World Medical Association and was approved by the Institutional Ethical Board of University Hospital Motol, Prague, Czech Republic on 17 June 2020.

\subsection{Procedures}

First, all patients completed a standardized St Marks Incontinence Score (SMIS) questionnaire and underwent HRAM. Anal sphincter pressure at rest, maximal pressure increment and endurance were measured. Then, they received individual physiotherapy from the same skilled physiotherapists 1 time per week, 10 times in total. Patients were advised to perform self-treatment at least 4 times per week reporting the length and frequency of self-treatment in a diary. After the 10 weeks HRAM and SMIS were performed again. Subsequently, the patients underwent three months self-treatment period reporting the exercise in the diary and the final HRAM and SMIS were recorded once more after the 3 months self-treatment period.

\subsection{Assessment methods}

\subsubsection{High resolution anorectal manometry (HRAM)}

HRAM was performed using GI Solar system (Medical Measurement Systems $=$ MMS) by the same skilled gastroenterologist with more than 10 years HRAM experience. Before the examination itself, the patient needs to be emptied. During the assessment the patient was laying on the left side with his legs flexed. Calibrated and lubricated water perfused catheter with 8 circumferential sensor elements was gently inserted into the rectum to a depth of about $6 \mathrm{~cm}$, with the most distal sensor remaining externally thus registering the atmospheric pressure and the most proximal sensor registering the pressures at the depth of the anal canal (Kang et al., 2015; Lee \& Bharucha, 2016).

The following 3 variables were measured:

1. Resting pressure mean is the average pressure $(\mathrm{mmHg})$ monitored in the anal canal for a period of 20 seconds (Lee \& Bharucha, 2016). The subjects were instructed not to perform any PF muscle contractions just to remain relaxed.

2. Maximal pressure increment: The subjects were instructed to squeeze and suck in the PF with maximum force and hold. The examiner verbally motivated the subject to perform maximum contraction for a period of 5 seconds. After 30 seconds of relaxation the same measurement was repeated (Carrington et al., 2014). The higher value was used for the statistical analysis. The maximal squeeze increment is calculated as the difference between maximum anal squeeze pressure and anal resting pressure (Noelting et al., 2012).

3. Maximal squeeze pressure endurance: The subjects were challenged to maintain the 
maximum contraction for a period of $20 \mathrm{sec}-$ onds. Standard protocols suggest to measure endurance over a period of 30 seconds (Carrington et al., 2014; Scott \& Carrington, 2020) but this was too challenging for our MS patients and therefore we reduced this period to 20 seconds. The examiner verbally motivated the patients to maintain maximum contraction informing them when the half of the required time $(10 \mathrm{~s})$ had elapsed and then five seconds before the end of the maneuver. For statistical analysis the average pressure from the monitored 20 seconds was used. A numerical value denoted as the area under the curve, calculated by the MMS program as the area between the markers indicating the beginning and end of the 20 second contraction, was also used for statistical analysis to evaluate the overall endurance ability.

\subsubsection{St. Mark's incontinence score}

The subjects completed the St. Mark's incontinence score (SMIS) three times (Maeda et al., 2008). At the start of the study before the first physiotherapy, after the 10 weeks of physiotherapy under the therapist's supervision and at the end of the study, i.e. after another 3 months of self-treatment. SMIS provides the information about the nature of incontinence, patient's need for lifestyle changes, the use of plugs or pads, the ability to delay defecation and the need to take any antidiarrheal medication (Norderval et al., 2019; Vaizey et al., 1999). SMIS is sensitive to subjective changes in ARD regardless the type of incontinence, age or sex of the patient (Maeda et al., 2008), and has been shown to be positively correlated with quality of life measures in patients experiencing incontinence (Roos et al., 2009).

\subsection{Rehabilitation procedures}

All participants patients underwent 10 individual therapies provided by an experienced physiotherapist. During each 60 minutes physiotherapy session soft tissue and mobilization techniques in the lumbopelvic area were applied to treat trigger points and joint blockages (Pedraza et al., 2014). Visceral therapy to treat any resistances in the abdominal cavity including vaginal and rectal manual treatment of muscle spasms was applied as well as any scar treatment in cases where needed. Assessment according to PERFECT scheme (Laycock \& Jerwood, 2001) defined patients with muscle weakness less than $2 / 5$ (manual muscle testing) who received electric PF muscle stimulation once a week, i.e. 10 times in total using Enraf - Nonius, Myomed 632 X device. Other patients used biofeedback anal probe to train pelvic flor activation. Postural correction was applied by the therapist following Dynamic Neuromuscular Stabilization (DNS) principles and protocols to improve core stability (Frank et al., 2013; Kobesova et al., 2016). All patients were instructed in PF contraction training, executing exercises in supine, sitting and standing postures, along with more challenging situations such as: coughing, squatting, jumping, lifting a load or jogging if able to perform such activities.

The patients were educated about PF anatomy and function. Using the PERFECT scheme (Laycock \& Jerwood, 2001) which evaluates PF power, number of squeezes the patient can perform and the endurance, individually tailored self-treatment protocol was defined for each patient consisting of the following parts:

1) Reeducation of the defecation act in patients with the constipation - increased position of lower extremities, voluntary relaxation of the PF with subsequent training of the targeted localized pressure to the rectum and abdominal massage (Cotterill et al., 2018; Khera et al., 2019).

2) Bowel drill/bladder drill - during urgency the individual pulls the $\mathrm{PF}$ in and squeezes the sphincters to stop the urgency and avoid the leaks (Booth et al., 2020).

3) Postural training according to DNS approach to achieve optimal trunk stabilization and intraabdominal pressure regulation (Frank et al., 2013; Kobesova et al., 2016). Training of PF squeeze in DNS exercise positions: supine, prone, sitting, standing, squatting, jumping and when lifting a load.

4) Stretching of hamstrings, adductors and tensor fascia lata muscle. Patients suffering from spasticity preformed progressive stretching for at least 10 minutes daily (Halabchi et al., 2017; Smania et al., 2010), patients with trigger points without spasticity were advised to stretch each group following trigger point treatment manuals (Majlesi \& Unalan, 2010; Yumpu.com, n.d.).

5) Patients were advised to perform dynamic physical activity 2-3 times a week and resistance training 2-3 times per week following physical activity guidelines for MS patients (Kim et al., 2019). 
Table 2

Comparison of SMIS scores and HRAM readings across supervised physiotherapy and self-treatment sessions. Values are Mean (Standard Deviation)

\begin{tabular}{lcccrr}
\hline Measure & $\begin{array}{c}\text { Baseline } \\
\text { mean (SD) }\end{array}$ & $\begin{array}{c}\text { Post supervised } \\
\text { treatment mean } \\
(\mathrm{SD})\end{array}$ & $\begin{array}{c}\text { Post self- } \\
\text { treatment } \\
\text { mean (SD) }\end{array}$ & Effect size $^{\mathrm{c}}$ & $P$ value $^{-}$ \\
\hline SMIS & $14.00(4.97)$ & $9.70(4.88)$ & $9.30(3.13)$ & 0.449 & $0.005^{*}$ \\
Resting pressure $^{\mathrm{a}}$ & $55.83(18.93)$ & $52.69(21.62)$ & $51.84(16.58)$ & 0.038 & 0.704 \\
Maximal pressure increment $^{\mathrm{a}}$ & $49.85(36.71)$ & $57.60(55.59)$ & $60.88(46.46)$ & 0.058 & 0.582 \\
Maximal pressure endurance $^{\mathrm{a}}$ & $36.41(33.30)$ & $46.89(44.38)$ & $49.95(52.81)$ & 0.055 & 0.529 \\
Area under curve $^{\mathrm{b}}$ & $230.0(233.6)$ & $520.8(423.5)$ & $501.9(532.0)$ & 0.248 & 0.107 \\
\hline
\end{tabular}

Note: HRAM = High resolution anal manometry. SMIS = St. Marks Incontinence Score.${ }^{\mathrm{a}}$ HRAM measures in $(\mathrm{mmHg}) .{ }^{\mathrm{b}} \mathrm{Area}$ under curve measures in $(\mathrm{mmHg} / \mathrm{s}) .{ }^{c}$ Effect size $=$ Partial eta squared $\left(\eta^{2}\right) .{ }^{*}$ Statistically significantly difference observed $(p<0.05)$.

6) Patients were advised to respect exhaustion and prefer shorter exercise sessions (10-15 minutes) several times (2-3 times) a day.

\subsection{Statistical analysis}

Descriptive statistics were calculated for all variables. Data are mean \pm standard deviation, unless otherwise stated. A one-way repeated measures analysis of variance (ANOVA) was used to determine whether there were differences in HRAM readings (resting pressure, maximal pressure increment, maximal pressure endurance) and SMIS scores over three months of supervised physiotherapy and three additional months of self-treatment. There were several outliers in the data, as assessed by boxplots. However, due to the small sample size, outliers were retained in the data; with no appreciable differences comparing results after modifying to larger or smaller than next closest values. Not all data was normally distributed for each time point, as assessed by Shapiro-Wilk's test ( $p>.05)$, however such data were not transformed, as ANOVAs are robust to non-normality. In data where sphericity was violated, Greenhouse-Geisser corrections were applied. Main effects were run for time, and post-hoc tests were conducted when necessary. Data analyses were conducted with the Statistical Package for the Social Sciences (SPSS version 27.0 for Mac; IMB Corp, Armonk, NY).

\section{Results}

Ten patients completed the entire study. Descriptive statistics are presented for the participants in Table 1, with all HRAM and SMIS data presented in Table 2. For the SMIS, the main effect of time showed a statistically significant difference

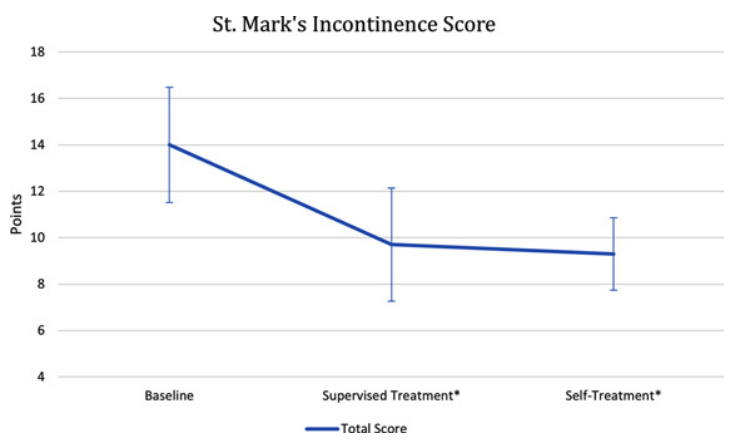

Fig. 1. St. Mark's Incontinence Scores values for all time measurements. *Significant difference observed from baseline, $p<0.05$.

between time points during the intervention period $\left(\mathrm{F}(2,18)=7.32, p=0.005\right.$, partial $\left.\eta^{2}=.45\right)$. Post -hoc analyses revealed a decrease in SMIS from baseline scores pre-intervention of $14.0 \pm 4.97$ points to $9.70 \pm 4.88$ points three months after supervised physiotherapy treatment sessions, a statistically significant decrease of 4.30 (95\% CI, 1.27 to 7.33 ) points, $p=0.007$, and another decrease in SMIS to $9.30 \pm 3.13$ points after three additional months of self-treatment, a statistically significant decrease of 4.70 (95\% CI, 0.58 to 8.83) points, $p=0.026$ compared with to baseline. There was no significant difference between the supervised and self-treatment time points (Fig. 1). The observed power, analyzed post-hoc, was 0.82 for the SMIS. For the HRAM, there were no statistically significant differences in time for any of the readings including: resting pressures $(\mathrm{F}(2,18)=0.358, p=0.70)$, pressure increment $(\mathrm{F}(2,18)=0.559, p=0.58)$, pressure endurance $(\mathrm{F}(1.28,11.55)=0.52, p=0.53)$, or area under the curve $(\mathrm{F}(1.24,11.18)=2.97, p=0.16)($ Table 2 and Fig 2). 

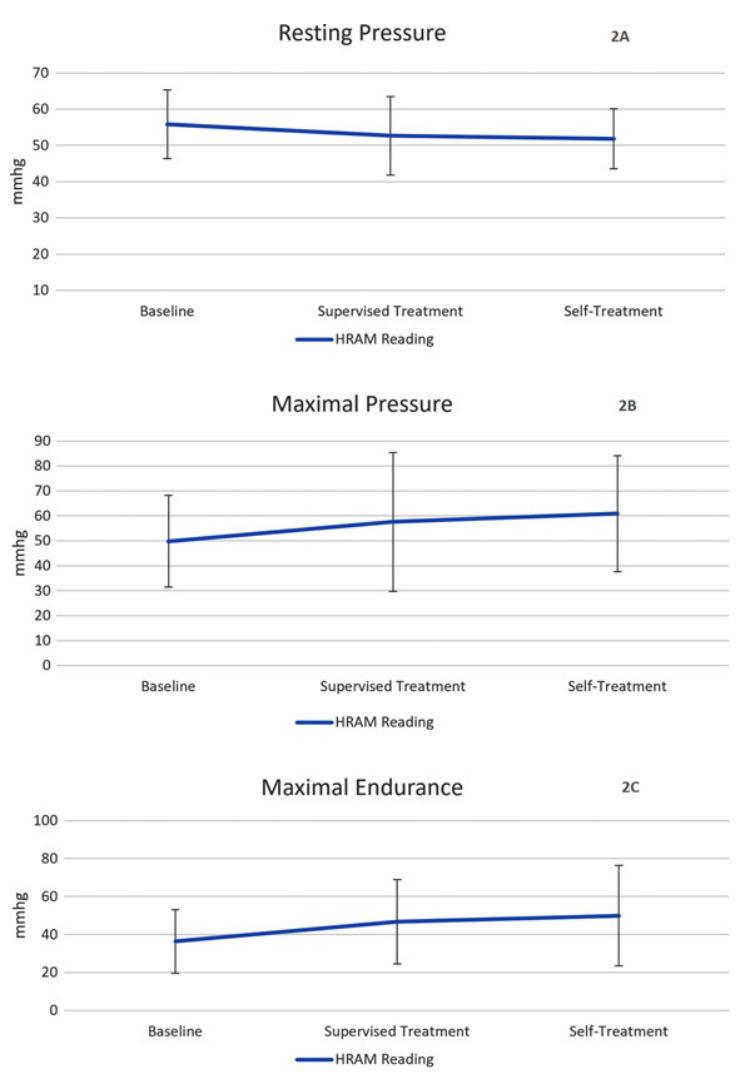

Fig. 2. 2A:Trend in resting pressure, 2B: Trend in maximum pressure, 2C: Trend in endurance increase over the monitored time. No significant differences noted from baseline.

\section{Discussion}

The results of this study demonstrate some initial benefits from an individualized rehabilitation program in MS patients suffering from ARD. Albeit fecal incontinence is frequent in the MS patient population (Chia et al., 1995; Hinds et al., 1990; Munteis et al., 2006; Pinter et al., 2015; Preziosi \& Emmanuel, 2009), this topic is still somewhat taboo (Preziosi et al., 2018) and patients are not adequately investigated and treated. Although half of our patients did not complete the whole study, they were all grateful that we actively asked about this delicate problem and offered targeted examinations, therapy, and advice on how to deal with such personally and socially devastating symptoms. This was also reflected in the subjective evaluation of the 10 patients who completed the study and reported a significant improvement. The effect of physiotherapy and feedback exercise on fecal incontinence of various origin was investigated by Norton et al (Nor- ton \& Cody, 2012) who analyzed the results of 21 studies. Although most papers publish uncontrolled studies, ARD symptoms improvement is reported in most of them. Norton concludes, that biofeedback, electrical stimulation and exercise may have a therapeutic effect, however, larger well-designed trials are needed to enable safe conclusions. The therapy effect was well proved in urinary incontinence (Kopańska et al., 2020; Mazur-Bialy et al., 2020; Nightingale, 2020). The PF muscles regulating stool continence and defecation are also involved in the physiological act of micturition, thus the similar exercise effect could be expected in both stool and urinary incontinence (Nusrat et al., 2012). The scientific literature mostly presents ARD functional assessment and treatment procedures in diagnoses other than MS (S. S. C. Rao et al., 2016). Albeit treatment principles may be the same in various types of ARD (S. S. C. Rao et al., 2016) not all types of conservative treatment were tested in MS patients and available studies are largely restricted to small case series (Nusrat et al., 2012; Preziosi et al., 2018). Exercise protocols, behavioral therapy and appropriate diet appears to be beneficial for patients with MS despite the lack of evidence (Nusrat et al., 2012; Preziosi et al., 2011, 2018). There are no precise therapeutic recommendations or guidelines, therefore the empirical treatment based on clinical symptoms and experience is mostly applied (Nusrat et al., 2012; Preziosi et al., 2018).

HRAM parameters measured in our study showed statistically insignificant improvements, likely due to the small sample who completed the study. Nevertheless, these findings are valuable because such research is lacking in the MS population. First, the resting pressure was measured. The normal range of anal pressures is relatively wide and dependent on sex and age, fluctuating between 32 and $88 \mathrm{mmHg}$ (Lee \& Bharucha, 2016; Noelting et al., 2012). In our study, the average resting pressure before intervention was $55.8 \mathrm{mmHg}$ decreasing slightly after the period of rehabilitation under the therapist's supervision to $52.7 \mathrm{mmHg}$ while the last measurement after 3 months of self-treatment remained at a similar level $(51.8 \mathrm{mmHg})$. Over $70 \%$ of resting anal pressure depends on the tone generated by the internal anal sphincter that is under the autonomic innervation (Keef \& Cobine, 2019). So, it is not surprising this variable demonstrated little change with rehabilitation that mainly addressed the skeletal muscle of the external anal sphincter (Fig. 2A). Also, the physiotherapist not only instructed patients how to activate the PF muscles, but relaxation training was another 
important aspect of rehabilitation. Perhaps the slight decrease in resting pressure resulted from patients' improved ability in relaxing the PF. Hyperactivity in the $\mathrm{PF}$ is associated with pelvic pain, urinary urgency, incontinence, defecatory dysfunction, and sexual symptoms; thus the relaxation procedures form an important part of PF rehabilitation (Aw et al., 2017).

The ability to increase anal pressure improved over the observed time period. Initial maximal pressure increment (maximal anal squeeze pressure minus anal resting pressure (Noelting et al., 2012)) of $49.85 \mathrm{mmHg}$ increased to 57.60 on the second measurement and even increased slightly to $60.88 \mathrm{mmHg}$ in the final measurement after 3 months of selftreatment. Such trend (Fig. 2B) is desirable, even though the difference was not great enough to reach statistical significance $(p=0.582)$ and none of the measured values (between $124 \pm 56$ and $174 \pm 81$ $\mathrm{mmHg}$ ) reached the defined norms for healthy populations based on age and gender (Oblizajek et al., 2019). Using the HRM apparatus in our gastroenterology center where this study was performed, 120 $\mathrm{mmHg}$ is considered to be normal for both genders, and incontinent patients typically demonstrate lower values (Ramage et al., 2019). The low maximum sphincter pressure in our MS cohort may have resulted from disturbed innervation of the external anal sphincter, however, the effort of the patient can also be a limitation (Lazarescu et al., 2009; Lee \& Bharucha, 2016). Therefore, it is necessary to motivate patients during the examination (Heinrich et al., 2013). The compliance and current emotional and cognitive state of our MS patients could also possibly influence the results of the measurements.

The last analyzed maneuver was the squeeze pressure endurance. According to Carrington et al. (Carrington et al., 2014), squeeze endurance has such a wide variation in healthy populations that this parameter is unlikely to be of diagnostic utility. Lee and Bharucha further describe that the clinical significance of low squeeze duration is unknown (Lee \& Bharucha, 2016). Additionally, there are variations in how researchers measure squeeze duration, thus recording different norms (Fox et al., 2004; Heinrich et al., 2013; Lee \& Bharucha, 2016; Noelting et al., 2012; Oblizajek et al., 2019; S. S. Rao et al., 1999). In our study we verbally motivated patients to maintain maximum contraction for 20 seconds using average pressure from the monitored 20 seconds for statistical analysis. The trend (Fig. 2C) of this parameter over the observed time was increasing but not reach- ing statistical significance $(p=0.107)$. The endurance squeeze pressure reflects mainly external sphincter function which can be addressed through training (Booth et al., 2020; Preziosi et al., 2011). Reduced endurance typically signifies external sphincter damage or dysfunction and it also describes fatigue (Fox et al., 2004). The endurance of the external anal sphincter is an important factor in maintaining fecal continence (Teng et al., 2018). Finally, for the pressure endurance maneuver we also calculated the area under the squeeze-duration curve, which is an indicator of the contraction endurance and decrease in muscle strength due to fatigue (Fox et al., 2004; Laycock \& Jerwood, 2001). From initial $230 \mathrm{mmHg} / \mathrm{s}$ this parameter desirably increased to $520.8 \mathrm{mmHg} / \mathrm{s}$ after the 3 months of guided intervention and then slightly dropped to 501.9 after another 3 months of selftreatment. The improving trend especially between the first and second measurement, however, have not reached statistical significance.

The only statistically significant improvement was identified in subjective perception of ARD by patients reported in the SMIS. The initial mean score of 14.00 points dropped to 9.70 after the series of supervised rehabilitation and more or less the same score ( 9.30 points) was reported after another three months of self-treatment. The subjective improvement was noted mainly in decreased incontinence both for solid and liquid stool and in the ability to defer the defection. Extensive research is available on ARD treatment, however, only limited number of studies describe patient's subjective perception of rehabilitation procedures on fecal incontinence in MS population specifically. Preziozi et al. (Preziosi et al., 2011) and Weisel (Wiesel, 2000) report positive effect of biofeedback behavioral therapy in MS patients. Posterior tibial nerve stimulation was identified as possibly effective for fecal incontinence treatment in MS patients (Sanagapalli et al., 2018) as well as sacral nerve stimulation which is discussed in the literature with inconsistent conclusions (Gulick \& Namey, 2012; Preziosi et al., 2018; Remmen \& Dindo, 2013). As far as exercise and physical activity, Gulick and Namey (Gulick \& Namey, 2012) state that structured exercise programs, aerobic training and fitness may bring improvement in bowel functioning, yet also warns how bowel and bladder symptoms may worsen with increased levels of physical activity. Pelvic floor muscle training combined with biofeedback resulted in subjective improvement of a MS patient with rectal prolapse (Sandalcidi, 2016) but this is only one case report. Still, the Consortium of Multiple Sclerosis 
Centers recommends exercise, physical therapy to increase general mobility, and core strengthening to treat bowel dysfunction (Newsome et al., 2017). Since SMIS positively correlates with patients quality of life (Roos et al., 2009) we consider the statistically significant improvement in our cohort to be of critical importance. From patient's perspective this maybe of more importance than any objective changes identified via HRAM.

Our treatment strategy involved patient's education, biofeedback, core stabilization exercises, sphincter contraction control training, stretching and relaxation procedures and manual treatment. While the number, frequency and length of treatment sessions were identical for all the subjects, the program itself, i.e. the combination and the length of various treatment procedures was individually tailored to each patient.

As far as the study limits, we acknowledge the lack of a control group and the small number of participants with only half of initially recruited participants completing the whole study. The MS population divers greatly in terms of clinical symptoms, speed of progression disease fluctuation, and the level of cognitive function which is decisive for rehabilitation coping and results. Perhaps, future studies should be done over a shorter period of time on more homogeneous sample of MS patients.

\section{Conclusions}

This study presents a positive overall effect of targeted physiotherapy programing on anorectal dysfunction in MS patients. Stool incontinence, measured through the SMIS, improved significantly. Despite nonsignificant HRAM results, the trend in data was favorable, with positive subjective perceptions suggesting that MS patients with ARD may benefit from the proposed complex rehabilitation approach. This rehabilitation approach may serve as the groundwork for future randomized controlled trials comparing rehabilitation strategies.

\section{Author contributions}

Martina Kovari: Conceptualization, Project administration, Methodology, Investigation, Data curation, Writing - original draft. Jan Stovicek: Conceptualization, Methodology, Investigation. Jakub Novak: Investigation, Data curation, Writing - review
\& editing. Michaela Havlickova: Project administration, Methodology, Investigation. Sarka Mala: Conceptualization, Project administration, Methodology, Investigation. Andrew Busch: Data curation, Software, Writing - review \& editing. Pavel Kolar: Conceptualization. Alena Kobesova: Conceptualization, Supervision, Writing - review \& editing, Funding acquisition.

\section{Conflict of interest}

There are no conflicts of interest to disclose.

\section{Ethical considerations}

The study conforms with The Code of Ethics of the World Medical Association and was approved by the Institutional Ethical Board of University Hospital Motol, Prague, Czech Republic on 17 June 2020.

\section{Funding}

This study was supported by the institutional research program Progress Q41 and by the foundation Movement without Help.

\section{References}

Aw, H. C., Ranasinghe, W., Tan, P. H. M., \& O’Connell, H. E. (2017). Overactive pelvic floor muscles (OPFM): Improving diagnostic accuracy with clinical examination and functional studies. Translational Andrology and Urology, 6(Suppl 2), S64-S67. https://doi.org/10.21037/tau.2017.05.41

Beer, S., Khan, F., \& Kesselring, J. (2012). Rehabilitation interventions in multiple sclerosis: An overview. Journal of Neurology, 259(9), 1994-2008. https://doi.org/10.1007/s00415-012-65774

Bols, E. M., Berghmans, B. C., Hendriks, E. J., de Bie, R. A., Melenhorst, J., van Gemert, W. G., \& Baeten, C. G. (2007). A randomized physiotherapy trial in patients with fecal incontinence: Design of the PhysioFIT-study. BMC Public Health, 7(1), 355. https://doi.org/10.1186/1471-2458-7-355

Booth, J., Bliss, D., \& International Continence Society Nursing Committee. (2020). Consensus statement on bladder training and bowel training. Neurourology and Urodynamics, 39(5), 1234-1254. https://doi.org/10.1002/nau.24345

Bywater, A., \& While, A. (2006). Management of bowel dysfunction in people with multiple sclerosis. British Journal of Community Nursing, 11(8), 333-334, 336-337, 340-341. https://doi.org/10.12968/bjen.2006.11.8.21665 
Carrington, E. V., Brokjaer, A., Craven, H., Zarate, N., Horrocks, E. J., Palit, S., Jackson, W., Duthie, G. S., Knowles, C. H., Lunniss, P. J., \& Scott, S. M. (2014). Traditional measures of normal anal sphincter function using highresolution anorectal manometry (HRAM) in 115 healthy volunteers. Neurogastroenterology \& Motility, 26(5), 625-635. https://doi.org/10.1111/nmo.12307

Chia, Y. W., Fowler, C. J., Kamm, M. A., Henry, M. M., Lemieux, M. C., \& Swash, M. (1995). Prevalence of bowel dysfunction in patients with multiple sclerosis and bladder dysfunction. Journal of Neurology, 242(2), 105-108. https://doi.org/10.1007/BF00887825

Cotterill, N., Madersbacher, H., Wyndaele, J. J., Apostolidis, A., Drake, M. J., Gajewski, J., Heesakkers, J., Panicker, J., Radziszewski, P., Sakakibara, R., Sievert, K.-D., Hamid, R., Kessler, T. M., \& Emmanuel, A. (2018). Neurogenic bowel dysfunction: Clinical management recommendations of the Neurologic Incontinence Committee of the Fifth International Consultation on Incontinence 2013. Neurourology and Urodynamics, 37(1), 46-53. https://doi.org/10.1002/nau. 23289

Fox, M., Schwizer, W., Menne, D., Stutz, B., Fried, M., \& Thumshirn, M. (2004). The physical properties of rectal contents have effects on anorectal continence: Insights from a study into the cause of fecal spotting on orlistat. Diseases of the Colon and Rectum, 47(12), 2147-2156. https://doi.org/10.1007/s10350-004-0739-0

Frank, C., Kobesova, A., \& Kolar, P. (2013). Dynamic neuromuscular stabilization \& sports rehabilitation. International Journal of Sports Physical Therapy, 8(1), 62-73.

Gosling, J., Plumb, A., Taylor, S. A., Cohen, R., \& Emmanuel, A. V. (2019). High-resolution anal manometry: Repeatability, validation, and comparison with conventional manometry. Neurogastroenterology \& Motility, 31(6), e13591. https://doi.org/10.1111/nmo.13591

Gulick, E., \& Namey, M. (2012). Bowel Dysfunction in Persons with Multiple Sclerosis. https://doi.org/10.5772/29317

Halabchi, F., Alizadeh, Z., Sahraian, M. A., \& Abolhasani, M. (2017). Exercise prescription for patients with multiple sclerosis; potential benefits and practical recommendations. BMC Neurology, 17(1), 185. https://doi.org/10.1186/s12883-0170960-9

Heinrich, H., Fruehauf, H., Sauter, M., Steingötter, A., Fried, M., Schwizer, W., \& Fox, M. (2013). The effect of standard compared to enhanced instruction and verbal feedback on anorectal manometry measurements. Neurogastroenterology and Motility: The Official Journal of the European Gastrointestinal Motility Society, 25(3), 230-237, e163. https://doi.org/10.1111/nmo.12038

Hinds, J. P., Eidelman, B. H., \& Wald, A. (1990). Prevalence of bowel dysfunction in multiple sclerosis. A population survey. Gastroenterology, 98(6), 1538-1542. https://doi.org/10.1016/0016-5085(90)91087-m

Kang, H. R., Lee, J.-E., Lee, J. S., Lee, T. H., Hong, S. J., Kim, J. O., Jeon, S. R., \& Kim, H. G. (2015). Comparison of High-resolution Anorectal Manometry With Water-perfused Anorectal Manometry. Journal of Neurogastroenterology and Motility, 21(1), 126-132. https://doi.org/10.5056/jnm 14025

Keef, K. D., \& Cobine, C. A. (2019). Control of Motility in the Internal Anal Sphincter. Journal of Neurogastroenterology and Motility, 25(2), 189-204. https://doi.org/10.5056/jnm18172
Khera, A. J., Chase, J. W., Salzberg, M., Thompson, A. J. V., \& Kamm, M. A. (2019). Systematic review: Pelvic floor muscle training for functional bowel symptoms in inflammatory bowel disease. JGH Open: An Open Access Journal of Gastroenterology and Hepatology, 3(6), 494-507. https://doi.org/10.1002/jgh3.12207

Kim, Y., Lai, B., Mehta, T., Thirumalai, M., Padalabalanarayanan, S., Rimmer, J. H., \& Motl, R. W. (2019). Exercise training guidelines for multiple sclerosis, stroke, and Parkinson's disease: Rapid review and synthesis. American Journal of Physical Medicine \& Rehabilitation, 98(7), 613-621. https://doi.org/10.1097/PHM.0000000000001174

Kobesova, A., Safarova, Marcela, R. M., \& Kolar, Pavel. (2016). Dynamic neuromuscular stabilization: Exercise in developmental positions to achieve spinal stability and functional joint centration. In Textbook of musculoskeletal medicine. Oxford University Press. http://www.tandfonline.com/toc/rwhi20/

Kopańska, M., Torices, S., Czech, J., Koziara, W., Toborek, M., \& Dobrek, E. (2020). Urinary incontinence in women: Biofeedback as an innovative treatment method. Therapeutic Advances in Urology, 12, 1756287220934359. https://doi.org/10.1177/1756287220934359

Krogh, K., \& Christensen, P. (2009). Neurogenic colorectal and pelvic floor dysfunction. Best Practice \& Research. Clinical Gastroenterology, 23(4), 531-543. https://doi.org/10.1016/j.bpg.2009.04.012

Laycock, J., \& Jerwood, D. (2001). Pelvic Floor Muscle Assessment: The PERFECT Scheme. Physiotherapy, 87(12), 631-642. https://doi.org/10.1016/S0031-9406(05)61108-X

Lazarescu, A., Turnbull, G. K., \& Vanner, S. (2009). Investigating and treating fecal incontinence: When and how. Canadian Journal of Gastroenterology, 23(4), 301-308.

Lee, T. H., \& Bharucha, A. E. (2016). How to Perform and Interpret a High-resolution Anorectal Manometry Test. Journal of Neurogastroenterology and Motility, 22(1), 46-59. https://doi.org/10.5056/jnm15168

Lensch, E., \& Jost, W. H. (2011). Autonomic Disorders in Multiple Sclerosis. Autoimmune Diseases, 2011. https://doi.org/10.4061/2011/803841

Maeda, Y., Parés, D., Norton, C., Vaizey, C. J., \& Kamm, M. A. (2008). Does the St. Mark's incontinence score reflect patients' perceptions? A review of 390 patients. Diseases of the Colon and Rectum, 51(4), 436-442. https://doi.org/10.1007/s10350007-9157-4

Majlesi, J., \& Unalan, H. (2010). Effect of treatment on trigger points. Current Pain and Headache Reports, 14(5), 353-360. https://doi.org/10.1007/s11916-010-0132-8

Marola, S., Ferrarese, A., Gibin, E., Capobianco, M., Bertolotto, A., Enrico, S., Solej, M., Martino, V., Destefano, I., \& Nano, M. (2016). Anal sphincter dysfunction in multiple sclerosis: An observation manometric study. Open Medicine (Warsaw, Poland), 11(1), 509-517. https://doi.org/10.1515/med-20160088

Mazur-Bialy, A. I., Kolomańska-Bogucka, D., Nowakowski, C., \& Tim, S. (2020). Urinary Incontinence in Women: Modern Methods of Physiotherapy as a Support for Surgical Treatment or Independent Therapy. Journal of Clinical Medicine, 9(4), E1211. https://doi.org/10.3390/jcm9041211

Motl, R. W., Sandroff, B. M., Kwakkel, G., Dalgas, U., Feinstein, A., Heesen, C., Feys, P., \& Thompson, A. J. (2017). Exercise in patients with multiple sclerosis. The Lancet Neurology, 16(10), 848-856. https://doi.org/10.1016/S1474-4422(17)30281-8 
Munteis, E., Andreu, M., Martinez-Rodriguez, J., Ois, A., Bory, F., \& Roquer, J. (2008). Manometric correlations of anorectal dysfunction and biofeedback outcome in patients with multiple sclerosis. Multiple Sclerosis (Houndmills, Basingstoke, England), 14(2), 237-242. https://doi.org/10.1177/ 1352458507082606

Munteis, E., Andreu, M., Téllez, M. J., Mon, D., Ois, A., \& Roquer, J. (2006). Anorectal dysfunction in multiple sclerosis. Multiple Sclerosis (Houndmills, Basingstoke, England), 12(2), 215-218. https://doi.org/10.1191/135248506ms $1254 \mathrm{oa}$

Newsome, S. D., Aliotta, P. J., Bainbridge, J., Bennett, S. E., Cutter, G., Fenton, K., Lublin, F., Northrop, D., Rintell, D., Walker, B. D., Weigel, M., Zackowski, K., \& Jones, D. E. (2017). A Framework of Care in Multiple Sclerosis, Part 2. International Journal of MS Care, 19(1), 42-56. https://doi.org/ 10.7224/1537-2073.2016-062

Nightingale, G. (2020). Management of urinary incontinence. Post Reproductive Health, 26(2), 63-70. https://doi.org/10. 1177/2053369120927112

Noelting, J., Ratuapli, S. K., Bharucha, A. E., Harvey, D. M., Ravi, K., \& Zinsmeister, A. R. (2012). Normal values for high-resolution anorectal manometry in healthy women: Effects of age and significance of rectoanal gradient. The American Journal of Gastroenterology, 107(10), 1530-1536. https://doi.org/10.1038/ajg.2012.221

Nordenbo, A. M., Andersen, J. R., \& Andersen, J. T. (1996). Disturbances of ano-rectal function in multiple sclerosis. Journal of Neurology, 243(6), 445-451. https://doi.org/10.1007/BF00900497

Norderval, S., Rydningen, M. B., Falk, R. S., Stordahl, A., \& Johannessen, H. H. (2019). Strong agreement between interviewobtained and self-administered Wexner and St. Mark's scores using a single questionnaire. International Urogynecology Journal, 30(12), 2101-2108. https://doi.org/10.1007/s00192019-03945-6

Norton, C., \& Cody, J. D. (2012). Biofeedback and/or sphincter exercises for the treatment of faecal incontinence in adults. The Cochrane Database of Systematic Reviews, 7, CD002111. https://doi.org/10.1002/14651858.CD002111.pub3

Nusrat, S., Gulick, E., Levinthal, D., \& Bielefeldt, K. (2012). Anorectal Dysfunction in Multiple Sclerosis: A Systematic Review. ISRN Neurology, 2012. https://doi.org/ $10.5402 / 2012 / 376023$

Oblizajek, N. R., Gandhi, S., Sharma, M., Chakraborty, S., Muthyala, A., Prichard, D., Feuerhak, K., \& Bharucha, A. E. (2019). Anorectal pressures measured with high-resolution manometry in healthy people-Normal values and asymptomatic pelvic floor dysfunction. Neurogastroenterology and Motility: The Official Journal of the European Gastrointestinal Motility Society, 31(7), e13597. https://doi.org/ 10.1111/nmo.13597

Pedraza, R., Nieto, J., Ibarra, S., \& Haas, E. M. (2014). Pelvic Muscle Rehabilitation: A Standardized Protocol for Pelvic Floor Dysfunction. Advances in Urology, 2014, e487436. https://doi.org/10.1155/2014/487436

Pinter, A., Cseh, D., Sárközi, A., Illigens, B., \& Siepmann, T. (2015). Autonomic Dysregulation in Multiple Sclerosis. International Journal of Molecular Sciences, 16, 16920-16952. https://doi.org/10.3390/ijms160816920

Preziosi, G., \& Emmanuel, A. (2009). Neurogenic bowel dysfunction: Pathophysiology, clinical manifestations and treatment.
Expert Review of Gastroenterology \& Hepatology, 3(4), 417 423. https://doi.org/10.1586/egh.09.31

Preziosi, G., Gordon-Dixon, A., \& Emmanuel, A. (2018). Neurogenic bowel dysfunction in patients with multiple sclerosis: Prevalence, impact, and management strategies. Degenerative Neurological and Neuromuscular Disease, 8, 79-90. https://doi.org/10.2147/DNND.S138835

Preziosi, G., Raptis, D. A., Storrie, J., Raeburn, A., Fowler, C. J., \& Emmanuel, A. (2011). Bowel biofeedback treatment in patients with multiple sclerosis and bowel symptoms. Diseases of the Colon and Rectum, 54(9), 1114-1121. https://doi.org/10.1097/DCR.0b013e318223fd7b

Ramage, L., Qiu, S., Yeap, Z., Simillis, C., Kontovounisios, C. Tekkis, P., \& Tan, E. (2019). Anorectal Manometry Versus Patient-Reported Outcome Measures as a Predictor of Maximal Treatment for Fecal Incontinence. 35(6), 8.

Rao, S. S. C., Bharucha, A. E., Chiarioni, G., Felt-Bersma, R., Knowles, C., Malcolm, A., \& Wald, A. (2016). Anorectal Disorders. Gastroenterology, S0016-5085(16)00175-X 10.1053/ j.gastro.2016.02.009. https://doi.org/10.1053/j.gastro.2016. 02.009

Rao, S. S., Hatfield, R., Soffer, E., Rao, S., Beaty, J., \& Conklin, J. L. (1999). Manometric tests of anorectal function in healthy adults. The American Journal of Gastroenterology, 94(3), 773783. https://doi.org/10.1111/j.1572-0241.1999.00950.x

Remmen, F., \& Dindo, D. (2013). [Fecal incontinence-A treatable problem!]. Therapeutische Umschau. Revue Therapeutique, 70(7), 403-406. https://doi.org/10.1024/00405930/a000425

Roos, A.-M., Sultan, A. H., \& Thakar, R. (2009). St. Mark's incontinence score for assessment of anal incontinence following obstetric anal sphincter injuries (OASIS). International Urogynecology Journal and Pelvic Floor Dysfunction, 20(4), 407-410. https://doi.org/10.1007/s00192-008-0784-7

Ruiz, N. S., \& Kaiser, A. M. (2017). Fecal incontinenceChallenges and solutions. World Journal of Gastroenterology, 23(1), 11-24. https://doi.org/10.3748/wjg.v23.i1.11

Sanagapalli, S., Neilan, L., Lo, J. Y. T., Anandan, L., Liwanag, J., Raeburn, A., Athanasakos, E., Zarate-Lopez, N., \& Emmanuel, A. (2018). Efficacy of Percutaneous Posterior Tibial Nerve Stimulation for the Management of Fecal Incontinence in Multiple Sclerosis: A Pilot Study. Neuromodulation: Journal of the International Neuromodulation Society, 21(7), 682-687. https://doi.org/10.1111/ner.12764

Sandalcidi, D. (2016). Pelvic Floor Muscle Strengthening Using Biofeedback for Treatment of Fecal Incontinence in a Patient with Multiple Sclerosis. https://www.semanticscholar.org/ paper/Pelvic-Floor-Muscle-Strengthening-Using-Biofeedba ck-Sandalcidi/a73a5cc1df03662ad76b8e87a985f02c9f3f8a67

Scott, S. M., \& Carrington, E. V. (2020). The London Classification: Improving Characterization and Classification of Anorectal Function with Anorectal Manometry. Current Gastroenterology Reports, 22(11), 55. https://doi.org/10.1007/s11894020-00793-Z

Smania, N., Picelli, A., Munari, D., Geroin, C., Ianes, P., Waldner, A., \& Gandolfi, M. (2010). Rehabilitation procedures in the management of spasticity. European Journal of Physical and Rehabilitation Medicine, 46(3), 423-438.

Teng, M., Kervinio, F., Moutounaïck, M., Miget, G., Charlanes, A., Chesnel, C., Le Breton, F., \& Amarenco, G. (2018). Review of pelvic and perineal neuromuscular fatigue: Evaluation and impact on therapeutic strategies. Annals 
of Physical and Rehabilitation Medicine, 61(5), 345-351. https://doi.org/10.1016/j.rehab.2018.06.006

Vaizey, C., Carapeti, E., Cahill, J., \& Kamm, M. (1999). Prospective comparison of faecal incontinence grading systems. Gut, 44(1), 77-80.

Waldron, D. J., Horgan, P. G., Patel, F. R., Maguire, R., \& Given, H. F. (1993). Multiple sclerosis: Assessment of colonic and anorectal function in the presence of faecal incontinence. International Journal of Colorectal Disease, 8(4), 220-224. https://doi.org/10.1007/BF00290311
Wiesel, P. H. (2000). Gut focused behavioural treatment (biofeedback) for constipation and faecal incontinence in multiple sclerosis. Journal of Neurology, Neurosurgery \& Psychiatry, 69(2), 240-243. https://doi.org/10.1136/jnnp.69.2.240

Yumpu.com. (n.d.). Ebooks-download-travell-simons-simonsmyofascial-pain-and-dysfunction-the-trigger-point-manualunlimited. Yumpu.Com. Retrieved June 23, 2021, from https://www.yumpu.com/en/document/view/63118429/ebooksdownload-travell-simons-simons-myofascial-pain-anddysfunction-the-trigger-point-manual-unlimited 